\title{
Rapid onset obesity with hypothalamic dysfunction, hypoventilation and autonomic dysregulation in children: a case series from Saudi Arabia
}

\author{
Gawahir Mukhtar ${ }^{1}$, Wadha Alotaibi ${ }^{2}$, Khalid Al-mobaireek ${ }^{3}$, Safa Eltahir ${ }^{1}$, Suhail Al-Saleh ${ }^{4}$ \\ 1 -Pediatric Pulmonary Division, King Fahad Medical City (KFMC), 2 -Pediatric Pulmonary and Sleep \\ Physician, Head of the Pulmonary Unit, KFMC, KSA 3-Pediatric Pulmonary Division, King Khalid \\ Hospital/ King Saud University , 4 -Division of Respiratory Medicine, Hospital for Sick Children, \\ University of Toronto, Toronto, Ontario, Canada
}

Corresponding author: Gawahir Mohamed Ahmed Mukhtar, E mail: jmukhtar@kfmc.med.sa, Mobile number 00966543484521

\begin{abstract}
Background: rapid onset of obesity with hypothalamic dysfunction, hypoventilation and autonomic dysregulation (ROHHAD), which was previously known as late-onset central hypoventilation with hypothalamic dysfunction, is a rare and complex syndrome that has been described in several case reports and series worldwide. Patients with ROHHAD are usually reported to be healthy prior to a characteristic rapid weight gain associated with autonomic and hypothalamic dysfunction and fatal central hypoventilation in the absence of mutations in the paired-like homeobox 2B (PHOX2B) gene. Case study: despite increased advancing knowledge of the disease course, the variable onset often results in delaying or missed diagnosis of ROHHAD syndrome which resulted in fatal cardiopulmonary arrest, central hypoventilation or impaired neurocognitive function. Here, we reported the first case series of ROHAAD syndrome from Saudi Arabia comprising four patients with different clinical manifestations. In this article we reported 4 cases of ROHHAD as a first serial cases from Arab area according to our Knowledge, with variable manifestations, but all sharing the rapid onset of obesity and hypoventilation, but case 1 her main striking feature was altered pain sensation and thirsty as autonomic dysregulation, while the second patient was twin 2 had pseudotumor cerebri and behavioral changes and her second twin was healthy, the third case also had behavioral changes and case 4 had obstructive sleep apnea. Conclusion: due to the overlap in symptoms and signs between ROHHAD and other well-characterized disease entities, multidisciplinary care with input from a center with expertise in ROHHAD was crucial to the successful management of patients with suspected ROHHAD syndrome. Furthermore, mortality associated with the high incidence of cardiopulmonary arrest may be prevented by early ventilatory support.
\end{abstract}

Key words: ROHHAD, children, Saudi Arabia, case series, PHOX2B gene,

\section{Case Series:}

\section{Case 1}

The patient in case 1 was a young girl who first presented at age of 7 years with a history of rapid weight gain over the previous 4 months combined with a cough and shortness of breath for the previous 2 months. At admission, she was found to suffer from nocturnal hypoxia, morning hypercapnia (Reached $\mathrm{SpCO} 268 \mathrm{mmHg}$ ), hypernatremia (Serum sodium $>160 \mathrm{mmol} / \mathrm{L}$ ) as well as central precocious puberty with luteinizing hormone releasing hormone levels in the normal range and a normal cortisol stress response. Furthermore, the patient did not report polydipsia and only drank water upon request.
She also had unstable blood pressure and impaired pain sensitivity, which was typical of autonomic dysregulation. The patient was seen by a member of the nephrology team regarding her symptoms of hypernatremia and unstable blood pressure and by the endocrinology team, for hypernatremia as well as obesity and precocious puberty.

Extended investigations, included serum and urine osmolality, as well as brain and abdominal computerized tomography (CT) scans were performed. The patient received intravenous 
fluids and was started on a low salt diet to treat her high blood pressure and hypernatremia and on a low-calorie diet to reduce her weight.

Abdominal and chest CT scans revealed a left supra renal tumor, which was identified as a ganglioneuroma and was resected successfully (Figure1).

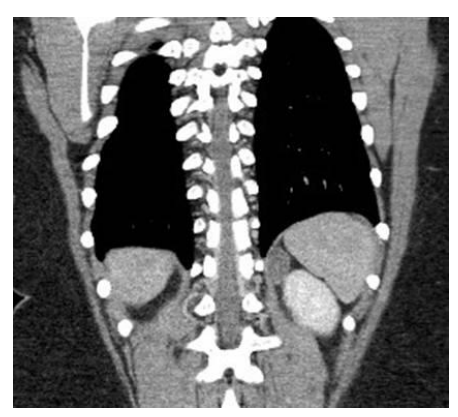

Figure1: abdominal and chest $\mathrm{CT}$ showed left supra renal tumor

In hospital, the child was started on bi-level positive airway pressure (Bi-PAP) ventilation. Echocardiography showed no evidence of pulmonary hypertension. Unfortunately, no tests for PHOX2B mutations were performed and the child was discharged on non-invasive ventilation at home. With good compliance and regular follow-up examinations for 3 years till his age became 10 years, the patient was discharged from the clinic.

\section{Case 2}

The patient in case 2 was an 8 -year-old female twin born as a result of in vitro fertilization with a birth weight of $1.7 \mathrm{~kg}$, her weight increased rapidly from the age of 3 , reached $106 \mathrm{~kg}$ just before she underwent bariatric surgery (Sleeve gastrectomy) at the age of 4 . Because of symptoms suggestive of obstructive sleep apnea, which started at the age of 3 years at the time of the onset of her weight gain, she underwent adenotonsillectomy at the age of 7 years. She presented with a history of progressive respiratory distress and continuous weight gain despite the sleeve gastrectomy combined with behavioral changes and aggressiveness. At the time of admission, the child's condition had deteriorated and she was transferred to the intensive care unit for mechanical ventilation for significant residual atelectasis and hypoventilation (Figure2). The patient was maintained on nocturnal Bi-PAP. She also had temperature dysregulation and hypothyroidism, although her serum sodium levels and blood pressure were normal. She was also diagnosed with pseudo tumor cerebri and a ventriculoperitoneal shunt was inserted after there was no improvement in the intracranial pressure with medication. Cardiac echocardiography showed no evidence of pulmonary hypertension and she tested negative for the PHOX2B gene mutation.The patient was discharged after stabilization on Bilevel Positive Airway Pressure (Bi-PAP). However, she was suffered from cardiopulmonary arrest at home and was transferred to hospital where she was intubated and ventilated for more than 1 month. The patient was moved to a hospital in Germany for further management. Here, the child underwent a tracheostomy and according to the family, she was now progressing well with a marked reduction in her weight.

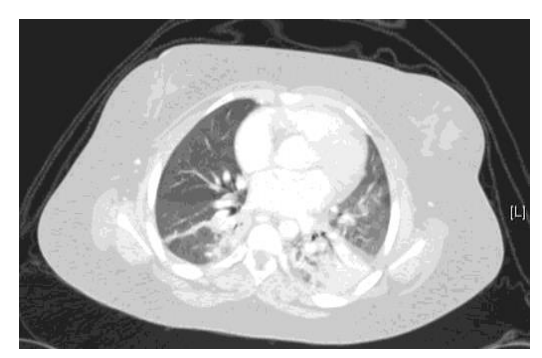

Figure2: CT chest showing atelectasis in the left lower lobe

\section{Case 3}

The patient in case 3 was a previously healthy 4year-old girl who, eight months before presentation, developed polyphagia with rapid weight gain of more than $12 \mathrm{~kg}$ (over a period of 2 months), polyuria and polydipsia, as well as snoring and sleep apnea. Early on the morning of 3 March 2013, she was found to be unresponsive at home by her parents with irregular breathing and cyanosis. Initially, she was rushed to a regional hospital with a Glasgow Coma Scale (GCS) score of $5 / 10$ and severe acidosis. A tracheostomy was performed due to failure of intubation and cardiopulmonary arrest. She developed seizures as well as blood pressure and temperature dysregulation. Subsequently, she 
was transferred to a tertiary institution for further investigations and management. Neurological evaluation and a complete work-up, included magnetic resonance imaging (MRI) and magnetic resonance spectroscopy, revealed a bilateral symmetrical cortical T2 hyper intensity and increased intensity in the basal ganglia, which was thought to be secondary to the chronic hypoxia. Other causes, included mitochondrial diseases and $\mathrm{N}$-methyl D-aspartate receptor antibody (NMDA) encephalitis, were ruled out.

Since the endocrine system is regulated by the hypothalamus and through hormones controls growth, energy and water balance, sexual maturation and fertility as well as the response to stress, we conducted investigations for endocrinopathies, such as diabetes insipidus (DI), because of the patient's history of polyuria and polydipsia; however, all tests were negative apart from low TSH. At admission, the patient had clinical evidence of hypoventilation and required ventilation via tracheostomy (Figure3). She tested negative for PHOX2B gene mutations associated with congenital hypoventilation syndrome but developed some sleep pattern and behavioral changes as well as signs of anxiety and aggressiveness.

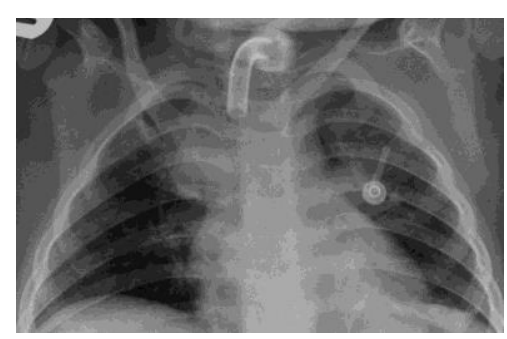

Figure3: CXR: showing tracheostomy tube and bilateral lower lobar and right upper lobe segmental atelectasis

\section{Case 4}

The patient in case 4 was a previously healthy 9year-old girl who presented with a 2-months history of polyphagia and rapid weight gain of more than $12 \mathrm{~kg}$, increased sweating and snoring, witness apnea and mouth breathing. The patient was admitted initially for investigations, but was found to be unresponsive in the early morning 2 days later. She had a GCS score of 5/10 with irregular breathing and cardiopulmonary arrest. The girl was intubated and transferred to the Pediatric Intensive Care Unit (PICU) for mechanical ventilation. Neurological evaluation and investigations, included brain MRI, showed pituitary microadenoma. All the endocrine hormones were within the normal range, except increased PTH and prolactin levels were detected. The patient was discharged, but was readmitted as an emergency 1 week later with seizures and behavioral changes. She was reintubated and investigations revealed that the seizures were related to hypoxia rather than epilepsy.

Repeated echocardiography was normal and there was no pulmonary hypertension. No abnormalities were identified in metabolic investigations and PHOX2B gene mutations were not detected. The patient underwent adenotonsillectomy after confirmation of adenotonsillar hypertrophy with significant clinical obstructive sleep apnea (Figure 4). Overnight pulse oximetry showed significant hypoxia and early morning capillary blood gases showed increased pCO2 $(78 \mathrm{mmHg})$. The patient was discharged on Bi-PAP during sleeping, but was re- admitted after 3 months with lethargy and seizures accompanied by increased pCO2 (95 $\mathrm{mmHg}$ ). After 1 month in the PICU, she was discharged on a home ventilator and monitored as an outpatient in the sleep clinic.

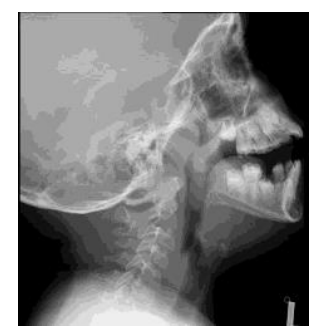

Figure 4: nasopharynx: Enlarged adenoid and palatine tonsils causing narrowing of the nasopharyngeal airway

\section{DISCUSSION}


Table 1: patients demographic and presentations

\begin{tabular}{|c|c|c|c|c|}
\hline Criteria & Case 1 & Case 2 & Case 3 & Case 4 \\
\hline $\begin{array}{l}\text { Age at onset of } \\
\text { weight gain (years) }\end{array}$ & 6-7 & 3-4 & $3-4$ & $8-9$ \\
\hline $\begin{array}{l}\text { Hypothalamic } \\
\text { dysfunction }\end{array}$ & $\begin{array}{l}\text { Precocious } \\
\text { puberty }\end{array}$ & Hypothyroidism & $\begin{array}{l}\text { Polyphagia; } \\
\text { clinical symptoms } \\
\text { of DI in the form of } \\
\text { polyuria and } \\
\text { polydipsia }\end{array}$ & $\begin{array}{l}\text { Polyphagia; } \\
\text { increased PTH and } \\
\text { prolactin }\end{array}$ \\
\hline Hypoventilation & Present & $\begin{array}{l}\text { Present with } \\
\text { cardiopulmonary } \\
\text { arrest }\end{array}$ & $\begin{array}{l}\text { Present with } \\
\text { cardiopulmonary } \\
\text { arrest }\end{array}$ & $\begin{array}{l}\text { Present with } \\
\text { cardiopulmonary } \\
\text { arrest }\end{array}$ \\
\hline $\begin{array}{l}\text { Autonomic } \\
\text { dysfunction }\end{array}$ & $\begin{array}{l}\text { Unstable blood } \\
\text { pressure and } \\
\text { impairment of } \\
\text { pain sensation and } \\
\text { thirst }\end{array}$ & Thermal instability & Thermal instability & Excessive sweating \\
\hline $\begin{array}{ll}\text { Water } & \text { and } \\
\text { electrolytes }\end{array}$ & $\begin{array}{l}\text { No polydipsia } \\
\text { Hypernatremia }\end{array}$ & Normal electrolytes & $\begin{array}{l}\text { Polyuria and } \\
\text { polydipsia; } \\
\text { normal electrolytes }\end{array}$ & Normal electrolytes \\
\hline $\begin{array}{ll}\text { Neural } & \text { crest } \\
\text { tumors }\end{array}$ & $\begin{array}{l}\text { Suprarenal } \\
\text { ganglioneuroma }\end{array}$ & Not found & Not found & Not found \\
\hline PHOX2B & Not done & Negative & Negative & Negative \\
\hline $\begin{array}{l}\text { Behavioral } \\
\text { changes }\end{array}$ & Not found & Present & Present & Present \\
\hline $\begin{array}{l}\text { Obstructive sleep } \\
\text { apnea (OSA) }\end{array}$ & Not detected & Present & Present & Present \\
\hline
\end{tabular}

Although approximately 80 cases of ROHHAD had been reported in the literature to date, the etiopathogenesis of this syndrome remains unclear. Here, we reported the first case series of ROHHAD syndrome from Saudi Arabia.ROHHAD was diagnosed according to the following criteria: 1- rapid onset obesity and alveolar hypoventilation starting after the age of 18 months; 2- evidence of hypothalamic dysfunction, as defined by one or more of the following findings: rapid onset obesity, hyperprolactinemia, central hypothyroidism, dysregulated water balance, failed $\mathrm{GH}$ stimulation test, corticotrophin deficiency, or altered onset of puberty (delayed or precocious) and 3) - absence of PHOX2B gene mutation (to rule out congenital central hypoventilation syndrome CCHS) ${ }^{(1)}$.

The rapid onset of obesity in the first 10 years of life (mean age, 3 years) was the most characteristic manifestation observed in the patients reported in this series; this is in accordance with a previous report ${ }^{(2)}$.Alveolar hypoventilation can be associated with different cardiac and respiratory diseases; therefore, lung and heart diseases as well as neurological or metabolic diseases must be ruled out before central hypoventilation can be diagnosed (3). Serious episodes of hypoventilation were seen in all the cases in this series with cardiopulmonary arrest in Cases 2, 3 and 4. Cases 1- 3 were currently being maintained on non-invasive ventilation at home, while the patient in case 4 was on a home ventilator.Respiratory evaluation should be performed for assessment of spontaneous breathing during varying levels of typical daily activities among children (e.g. playing, running, and eating). Assessment of the child's response to endogenous and exogenous hypercarbia, hypoxemia and hyperoxia challenges while, awake should also be carried 
out in addition to assessment of responses to endogenous challenges while asleep under various conditions (e.g. sitting up, supine, at sleep onset and during sleep). Cardiac evaluation should be focused on detection of any signs of cor pulmonale or right ventricular hypertrophy, which may develop with chronic hypoventilation, and detection of prolonged sinus pause or a systole that might require a cardiac pacemaker ${ }^{(4)}$. No signs of cardiac disease were detected in the repeated echocardiography performed in all our patients. Furthermore, metabolic screening was negative in all four cases, despite strong suspicions of metabolic disease in case 3 .

Congenital central hypoventilation syndrome should be ruled out by testing for PHOX2B gene mutations. In this series, cases 2, 3 and 4 tested negative for mutations in this gene; unfortunately, the test was not done for case 1).The hypothalamic dysfunction that constitutes part of the ROHHAD syndrome could be due to congenital malformation, trauma, hypothalamic tumors and Langerhans cell histocytosis; therefore, radiological imaging of the central nervous system is necessary to distinguish the cause. In case 4, brain MRI showed microadenoma for neurological follow- up, while in case 3, brain MRI revealed some changes that could be explained by the chronic hypoxia. This patient was investigated extensively for neurological diseases, included NMDA receptor antibody encephalitis, and metabolic diseases, included those with mitochondrial causes were tested by muscle biopsy and detection of mitochondrial electron transport chain enzymes with negative results obtained. Case 1 underwent precocious puberty and Case 2 had hypothyroidism, while no hormonal disturbances except polyphagia were detected in cases 3 and 4 . Despite the presence of clinical symptoms of DI (polyuria and polydipsia), case 3 tested negative for this disorder. Case 4 had increased PTH and prolactin levels.

Autonomic dysfunction in ROHHAD syndrome can be manifested as light-nonresponsive pupils, impaired gastro-intestinal motility (constipation), body temperature disorders (hypothermia, hyperthermia), sweating disorders and reduced sensitivity to pain. The autonomic manifestations in our cases were variable. Case 1 had unstable blood pressure, clear impairment of pain sensitivity and abnormal thirst, while cases 2 and 3 had thermal instability and case 4 had excessive sweating. Diagnosis of ROHHAD was based on the clinical presentation and clinical course, which should be evaluated in a cooperative consultation by experts in the fields of respiratory, endocrine, and autonomic medicine (3). Some children had obstructive sleep apnea due to blocked air flow during sleep; this may be witness apnea or snoring during sleep. Because obstructive sleep apnea was common in obese children, a connection to the ROHHAD syndrome might be delayed, as in case 4 . Difficulties in the diagnosis of ROHHAD syndrome can be attributed to the frequency of hyperphagia as a cause of obesity, as in cases 3 and 4. There was some overlap between ROHHAD and other syndromes, such as Prader Willi syndrome, included morbid obesity, hyperphagia, sleep apnea and mental retardation; therefore, these syndromes must be considered in the differential diagnosis ${ }^{(5)}$. In our case series, the absence of the characteristic clinical features allowed us to rule out syndromic obesity.Patients with suspected ROHHAD syndrome should be screened for neural crest tumor since these are present in approximately $33 \%$ of these patients. In this series, a left supra renal tumor was identified and resected successfully in case 1 . The gene responsible for ROHHAD had not yet been identified and there was currently no genetic test available for a definitive diagnosis of this syndrome. Patwari et al. reported monozygotic twins, one with ROHHAD, while the other twin was not affected, indicating that autoimmune or epigenetic factors are not causative factors in ROHHAD syndrome ${ }^{(6)}$. In accordance with this, case 2 in our series was also a dizygotic twin whose twin (a boy) was healthy and unaffected by the syndrome. Sleep disturbances and obstructive sleep apnea are common problems among obese children that can delay the diagnosis of ROHHAD. In our series, cases 2- 4 had significant obstructive sleep apnea, with adenotonsillectomy performed in cases 2 and 4.Behavioural problems, psychosis, mental retardation, mood and personality changes have been defined as part of the ROHHAD syndrome and were reported in our cases 2- 4 Similarly, 
Dhondt $\boldsymbol{e t}$ al. reported cases of ROHHAD with sleep disturbances and narcolepsy ${ }^{(7)}$. Table 1 showing the demographic and presentations for the 4 cases.

\section{CONCLUSION}

Early recognition by the clinician of a child with rapid and dramatic weight gain and investigations for hypothalamic dysfunction, hypoventilation, autonomic dysfunction and tumors of neural crest origin are critical in achieving a diagnosis of ROHHAD syndrome; however, this is complicated by the overlap with other wellcharacterized disease entities.

Multidisciplinary care with input from a center with expertise in ROHHAD is crucial to the successful management of these patients. This team should include primary care physicians, pulmonologists, endocrinologists, cardiologists, gastroenterologists, neurologists, ophthalmologists, psychologists, psychiatrists, respiratory therapists, intensivists, otolaryngologists, and surgeons, as well as nurses, social workers, speech and language therapists, and special education teachers, all working together with the child and the family to optimize care and quality of life.

Mortality associated with the high incidence of cardiopulmonary arrest may be prevented by early ventilatory support.

\section{REFERENCES}

1. Ize-Ludlow D1, Gray JA, Sperling MA et al .(2007): Rapid-onset obesity with hypothalamic dysfunction, hypoventilation, and autonomic dysregulation presenting in childhood. Pediatrics, 120: 179-188.

\section{Rand CM, Patwari PP, Rodikova EA} et al. (2011): Rapid-onset obesity with hypothalamic dysfunction, hypoventilation, and autonomic dysregulation: analysis of hypothalamic and autonomic candidate genes. Pediatr. Res., 70:375-383.

\section{Bougneres $\mathbf{P}$, Pantalone L, Linglart A} et al.(2008): Endocrine manifestations of the rapid-onset obesity with hypoventilation, hypothalamic, autonomic dysregulation, and neural tumor syndrome in childhood. J. Clin. Endocrinol. Metab., 93:3971-3980.

4. De Pontual L1, Trochet D, Caillat-Zucman $S$ et al. (2008): Delineation of late onset hypoventilation associated with hypothalamic dysfunction syndrome. Pediatr Res., 64:689-694.

5. Abaci A, Catli G, Bayram E et al. (2013): A cases of rapid-onset obesity with hypothalamic dysfunction, hypoventilation, autonomic dysregulation, and neural crest tumor: ROHHADNET syndrome. Endocr. Pract., 19:1216.

6. Patwari PP, Rand CM, Berry-Kravis EM et al. (2011): Monozygotic twins discordant for ROHHAD phenotype. Pediatrics, 128:711-715.

7. Dhondt K, Verloo P, Verhelst H et al. (2013): Hypocretin-1 Deficiency in a Girl With ROHHAD Syndrome. Pediatrics, 132:788-792. 